\title{
Modelling, Simulation and Optimization of n-p-n-p Silicon Multilayer Solar Cells
}

\author{
Abdellatif Bouzidi*, Ahmed S. Bouazzi, Mosbah Amlouk \\ Unité de Physique des Dispositifs à Semi-Conducteurs, Faculté des Sciences de Tunis, Le Belvédère, Tunis, Tunisia \\ Email: "abdellatif.bouzidi@isi.rnu.tn
}

Received February 10, 2012; revised March 1, 2012; accepted May 9, 2012

\begin{abstract}
We simulate the conception parameters of a model of a silicon multilayer solar cell. The cell is composed by four layers of opposite conductivities forming three junctions inside the cell. The electric contacts are tailored vertically to collect the minority carrier generated under illumination. We developed the equations giving the output power, the fill factor and the efficiency of the cell, taking into account the series resistances of each layer. We optimized, using MATLAB software, the thicknesses of the layers, the impurity concentration level and the distance between the electric contacts. We showed that the optimized photovoltaic structure, with the silicon properties published at the Ioffe institute website, gives an efficiency of $20.66 \%$. The n-p-n-p silicon cell delivers a short circuit current $I_{c c}=45.3 \mathrm{~mA} / \mathrm{cm}^{2}$, an open circuit voltage $V_{o c}=0.746 \mathrm{~V}$ and an output power of $28.5 \mathrm{~mW} / \mathrm{cm}^{2}$. The corresponding fill factor is $F F=84.29 \%$.
\end{abstract}

Keywords: Solar Cell; Multilayer; Conception; Optimization

\section{Introduction}

Nowadays, multilayer solar cells occupy a major place in the field of photovoltaic conversion of solar energy, and they are extensively studied [1-4]. These new structures have the advantage of a better collection of the minority carriers generated by the light near the many depletion regions tailored in series inside the cell, each created electron-hole pair can meet, before recombining, a space charge region near the place where it is created.

Considerable research effort, both theoretically and experimentally, has been made to enhance efficiency of multilayer silicon solar cell. K. Brecl et al. [5] have developed an extended Ebers-Moll model for the examination of the physical parameters of the multilayer cell. They calculate the current-voltage characteristics of floating junction solar cells. They showed that the photocurrent density produced by an n-p-n-p multilayer solar cell with correspondent layers thicknesses of $0.5 \mu \mathrm{m}, 5.5 \mu \mathrm{m}, 0.4$ $\mu \mathrm{m}$ and $30 \mu \mathrm{m}$ respectively, is equal to $28.19 \mathrm{~mA} / \mathrm{cm}^{2}$ using the global AM1.5 solar spectrum.

In a previous work [6], we simulate and optimize the photocurrent densities in a model of an n-p-n-p type thin film multilayer silicon solar cell for space applications. The equations giving the photocurrent density in each layer of the cell have been developed. We showed that, under the optimum conditions, the n-p-n-p silicon multi-

${ }^{*}$ Corresponding author. layer cell delivers a photocurrent density of $46.28 \mathrm{~mA} / \mathrm{cm}^{2}$. This density of photocurrent represents the short-circuit current $I_{c c}$ that will be used to simulate the $I-V$ curves. The optimal thicknesses of the layers define, depending of the impurity concentration, the electrical series resistances of the equivalent electric circuit of the multilayer structure.

In another work [7], a new model of multilayer solar cell has been presented; the cell is composed by four layer of opposite conductivity including a frontal layer of n-type $6 \mathrm{H}-\mathrm{SiC}$ to absorb high energy photons and a back layer of p-type $\mathrm{Si}_{0.8} \mathrm{Ge}_{0.2}$ to absorb low energy photons of the solar spectrum. The tow other layers are p-type silicon layer and n-type silicon layer. The optical properties of the semiconductor materials used in this numerical simulation have been taken from the Ioffe institute website [8]. We showed that, under the optimum conditions, such optimized multilayer solar cell could deliver a photocurrent density of $53.5 \mathrm{~mA} / \mathrm{cm}^{2}$.

\section{Optimization of the n-p-n-p Silicon Multilayer Solar Cell}

After the optimization of the photocurrent densities produced by the multilayer solar cell by optimizing the layers thicknesses [6], we are interesting in the optimization of the output power and the efficiency of the cell.

An energy band diagram of the multilayer n-p-n-p solar cell is giving below (Figure 1). The total thickness of 
the cell is taken equal $100 \mu \mathrm{m}$ for mechanical rigidity.

The optimal abscissas were [6]: $d_{1}=0.1 \mu \mathrm{m}, d_{11}=0.2$ $\mu \mathrm{m}, d_{2}=13 \mu \mathrm{m}, d_{22}=13.1 \mu \mathrm{m}, d_{3}=82 \mu \mathrm{m}$ and $d_{33}=$ $82.1 \mu \mathrm{m}$.

The optimal photocurrent densities, as shown in Figure 1, are:

$J p d_{1}=5.3 \mathrm{~mA} / \mathrm{cm}^{2}, J n d_{11}=23.8 \mathrm{~mA} / \mathrm{cm}^{2}, J n d_{2}=6.9$ $\mathrm{mA} / \mathrm{cm}^{2}, J p d_{22}=5.4 \mathrm{~mA} / \mathrm{cm}^{2}, J p d_{3}=2.2 \mathrm{~mA} / \mathrm{cm}^{2}$, and $\operatorname{Jnd}_{33}=0.23 \mathrm{~mA} / \mathrm{cm}^{2}$, and the photocurrent densities dues to the space charge regions are:

$J s c r_{1}=2.4 \mathrm{~mA} / \mathrm{cm}^{2}, J s c r_{2}=0.045 \mathrm{~mA} / \mathrm{cm}^{2}$ and $J_{s c r}=$ $0.003 \mathrm{~mA} / \mathrm{cm}^{2}$.

The total photocurrent density produced by the optimized n-p-n-p multilayer silicon solar cell is then JTot equal to $46.42 \mathrm{~mA} / \mathrm{cm}^{2}$.

These densities of photocurrent determine the current produced by each junction $I_{1}, I_{2}$ and $I_{3}$ in the equivalent electric circuit giving in Figure 2. The abscissas of the layers, with the impurity concentration, give the series electrical resistances $R 1, R 2, R 3$ and $R 4$.
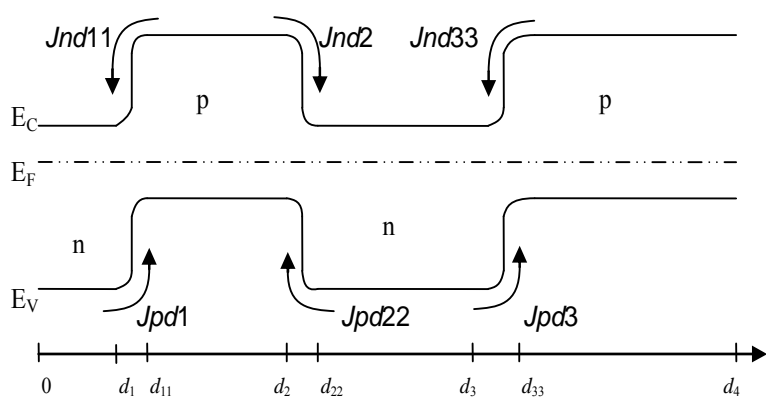

Figure 1. Energy band structure diagram of the multilayer silicon solar cell. The six arrows show the minority carriers path that produces the photocurrent.

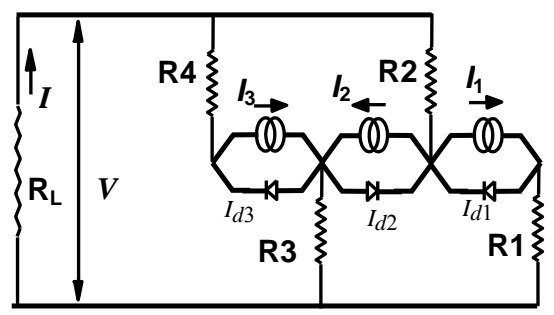

Figure 2. The equivalent electric circuit of the multilayer structure. The arrows show the photocurrent created at each junction.

$R_{L}$ is the charge resistance and $I d_{1}, I d_{2}$ and $I d_{3}$ are the diodes current.

The voltage $V$ can be calculated using three ways:

$$
V_{1}=\frac{K T}{q} \log \left(1+\frac{I d_{1}}{I_{0}}\right)-R_{1}\left(I_{1}-I d_{1}\right)-R_{2}\left(I_{1}+I_{2}\right)
$$

$V_{2}=\frac{K T}{q} \log \left(1+\frac{I d_{2}}{I_{0}}\right)-R_{2}\left(I_{1}+I_{2}\right)-R_{3}\left(I_{2}+I_{3}-I d_{2}-I d_{3}\right)$

$$
V_{3}=\frac{K T}{q} \log \left(1+\frac{I d_{3}}{I_{0}}\right)-R_{4} I_{3}-R_{3}\left(I_{2}+I_{3}-I d_{2}-I d_{3}\right)
$$

where $I_{0}$ is the saturation current of the diodes, and is giving by:

$$
I_{0}=q\left(\frac{p_{n} D_{p}}{L_{p}}+\frac{n_{p} D_{n}}{L_{n}}\right)
$$

$D_{p}$ and $D_{n}$ are respectively the diffusion coefficient of the holes and the electrons; $L_{p}$ and $L_{n}$ are respectively the diffusion length of the holes and electrons; $p_{n}$ and $n_{p}$ are the minority carrier concentration (holes and electrons respectively).

The output voltage of the cell is:

$$
V=\frac{\left(V_{1}+V_{2}+V_{3}\right)}{3}
$$

The output current is giving by:

$$
I=\left(I_{1}+I_{2}+I_{3}\right)-\left(I d_{1}+I d_{2}+I d_{3}\right)
$$

where:

$$
\begin{aligned}
& I_{1}=J p d 1+J s c r 1+J n d 11 \\
& I_{2}=J n d 2+J s c r 2+J p d 22 \\
& I_{3}=J p d 3+J s c r 3+J n d 33
\end{aligned}
$$

$I_{d 1}, I_{d 2}$ and $I_{d 3}$ are the diodes currents, they depends of the charge resistance $R_{L}$.

The series resistances of each layer are giving by [9]:

$$
\begin{gathered}
R_{1}=\rho_{n} \frac{L^{2}}{2 d_{1}} \\
R_{2}=\rho_{p} \frac{L^{2}}{2\left(d_{2}-d_{1}\right)} \\
R_{3}=\rho_{n} \frac{L^{2}}{2\left(d_{3}-d_{2}\right)} \\
R_{4}=\rho_{p} \frac{L^{2}}{2\left(d_{4}-d_{3}\right)}
\end{gathered}
$$

where, $\rho_{n}$ and $\rho_{p}$ are respectively the resistivity of the n-type layer and the p-type layer. $L$ is the distance between the n-contact and the p-contact (see Figure 3).

We introduced all the developed equations into MATLAB software to simulate under AM0 solar spectrum the $I-V$ characteristics of the cell. The maximum output power $P_{\max }$ can be deduced and the efficiency will be giving by:

$$
\eta=\frac{P_{\max }}{0.136}
$$

The efficiency depends of the impurity concentration level giving the electrons and holes electrons properties 


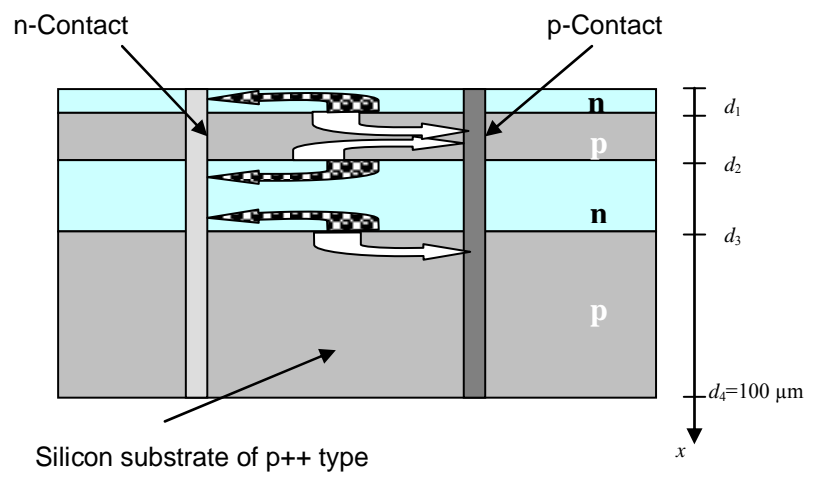

Figure 3. Cross section of the studied n-p-n-p multilayer silicon solar cell, the light enters from the n-side.

( $L_{n}, D_{n}$ and $L_{p}, D_{p}$ ). It depends also, of the layers thickness $\left(d_{1}, d_{2}-d_{1}, d_{3}-d_{2}\right.$ and $\left.d_{4}-d_{3}\right)$ in terms of the series resistances, and of the distance $L$ between n-contacts and p-contact. The optimization of the photovoltaic structure supposes three steps:

1) Optimization of the layers thickness;

2) Optimization of the impurity concentration;

3) Optimization of the distance between contacts.

\subsection{Optimization of Layers Thicknesses}

We begin by optimizing the layers thicknesses defined by the abscissas $d_{1}, d_{2}, d_{3}$ and $d_{4}$. We computed the efficiency of the n-p-n-p silicon solar cell by varying all the abscissas in a three dimensional space $d_{1}, d_{2}$ and $d_{3}$. The abscissa $d_{4}$ is fixed equal to $100 \mu \mathrm{m}$ which represents the total cell thickness. The impurity concentration is taken equals $10^{17} \mathrm{~cm}^{-3}$ in all the photovoltaic structure to ensure. As an illustration, Figure 4 gives the efficiency of the n-p-n-p solar cell versus the first and the second layer abscissa.

We can see that the efficiency of the cell decreases when $d_{1}$ and $d_{2}$ increases.

The program of simulation written in MATLAB gives the three optimal abscissas and the maximum efficiency under the prefixed simulation conditions:

$$
d_{1 \text { opt }}=4 \mu \mathrm{m}, d_{2 \text { opt }}=10 \mu \mathrm{m}, d_{3 \text { opt }}=45 \mu \mathrm{m} \text { and } \eta_{\max }=
$$

$19.78 \%$. Many other combinations of layers thickness giving the same value of the efficiency are possible.

\subsection{Optimization of the Impurity Concentration}

Here we optimize the impurity concentration using the optimal values of layers thicknesses. We simulate, under AM0 solar spectrum and assuming $100 \mathrm{~cm} / \mathrm{s}$ surface recombination velocity, the $I-V$ characteristics of the n-p-n-p solar cell for different values of the impurity concentration going from $10^{5} \mathrm{~cm}^{-3}$ to $10^{19} \mathrm{~cm}^{-3}$.

The electrical properties and the recombination parameters, depending of the impurity concentration were taken from Ioffe institute website [7] and given in Table 1.

We reported in Table 2 the results of simulation corresponding to the different values of the impurity concentration.

The efficiency of the simulated cells varies from $2.53 \%$ for an impurity concentration of $10^{15} \mathrm{~cm}^{-3}$ to $21.09 \%$ for an impurity concentration of $10^{18} \mathrm{~cm}^{-3}$. Figure 5 gives the $I-V$ characteristic corresponding to an impurity concentration of $10^{5} \mathrm{~cm}^{-3}$ (Figure 5(a)) and $10^{18} \mathrm{~cm}^{-3}$ (Figure 5(b)).

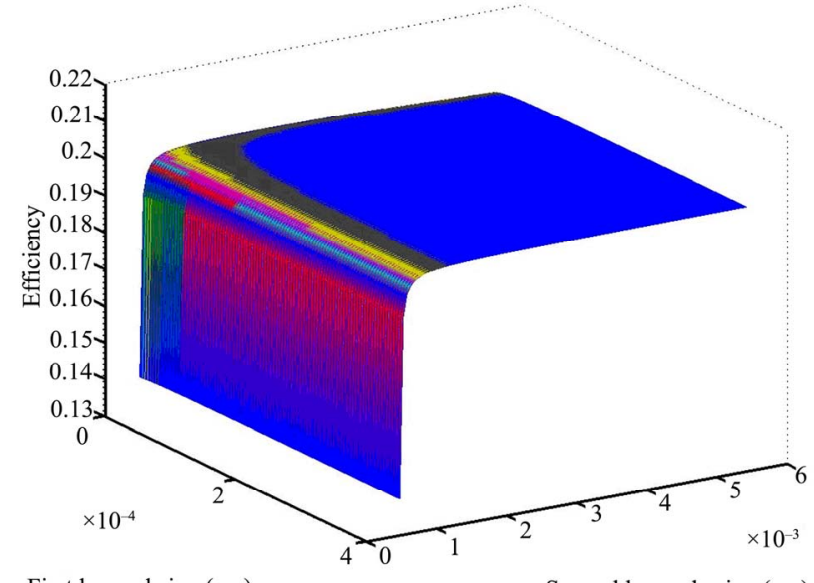

First layer absissa(cm)

Second layer abscissa $(\mathrm{cm})$

Figure 4. Efficiency of the n-p-n-p solar cell versus the first and the second layer abscissa. The third layer abscissa $d 3$ is taken equal to $60 \mu \mathrm{m}$.

Table 1. Recombination parameters versus impurity concentration.

\begin{tabular}{|c|c|c|c|c|c|c|}
\hline & Impurity concentration $\left(\mathrm{cm}^{-3}\right)$ & $10^{15}$ & $10^{16}$ & $10^{17}$ & $10^{18}$ & $10^{19}$ \\
\hline \multirow{4}{*}{$\underset{\Xi}{\stackrel{\Xi}{\Xi}}$} & Lifetime $\tau_{n}(\mathrm{~s})$ & $1.8 \times 10^{-4}$ & $7.4 \times 10^{-5}$ & $2.5 \times 10^{-5}$ & $4 \times 10^{-6}$ & $1.1 \times 10^{-7}$ \\
\hline & Diffusion length $L_{n}(\mathrm{~cm})$ & 0.08 & 0.05 & $2.2 \times 10^{-2}$ & $4.3 \times 10^{-3}$ & $4.5 \times 10^{-5}$ \\
\hline & Diffusion coefficient $D_{n}\left(\mathrm{~cm}^{2} / \mathrm{s}\right)$ & 45.71 & 33.79 & 19.36 & 4.62 & 1.84 \\
\hline & Resistively $\rho_{n}(\Omega$-cm $)$ & 5 & 0.58 & 0.09 & $2.2 \times 10^{-2}$ & $5 \times 10^{-3}$ \\
\hline \multirow{4}{*}{$\underbrace{\stackrel{\Xi}{2}}_{0}$} & Lifetime $\tau_{p}(\mathrm{~s})$ & $1.9 \times 10^{-4}$ & $7 \times 10^{-5}$ & $1.5 \times 10^{-5}$ & $1.4 \times 10^{-6}$ & $5.3 \times 10^{-8}$ \\
\hline & Diffusion length $L_{p}(\mathrm{~cm})$ & 0.041 & 0.024 & 0.008 & $1.7 \times 10^{-3}$ & $2.2 \times 10^{-4}$ \\
\hline & Diffusion coefficient $D_{p}\left(\mathrm{~cm}^{2} / \mathrm{s}\right)$ & 8.84 & 8.23 & 4.26 & 2.06 & 0.91 \\
\hline & Resistively $\rho(\Omega$-cm $)$ & 14 & 1.8 & 0.22 & $4.5 \times 10^{-2}$ & $9 \times 10^{-3}$ \\
\hline
\end{tabular}


The efficiency of the n-p-n-p silicon multilayer solar cell corresponding to an impurity concentration of $10^{5}$ $\mathrm{cm}^{-3}$ is too week (less then $3 \%$ ). This is due to the lack of minority carriers to be generated under illumination.

The efficiency of the cell decreases with an impurity concentration of $10^{19} \mathrm{~cm}^{-3}$ and it doesn't exceed $17 \%$. This low efficiency is due to the high recombination possibility and the important values of layers resistances, which depends on the minority carriers electrical properties as giving above.

We can see from Table 2 that the maximum efficiency is obtained with an impurity concentration of $10^{18} \mathrm{~cm}^{-3}$. Thus, the optimal concentration is in proximity of $10^{18}$ $\mathrm{cm}^{-3}$. We computed our simulation using an impurity concentration level between $3 \times 10^{17} \mathrm{~cm}^{-3}$ and $5 \times 10^{18}$ $\mathrm{cm}^{-3}$. The optimal impurity concentration is obtained equals to $10^{18} \mathrm{~cm}^{-3}$.

\subsection{Optimization of the Distance between Electric Contacts}

In all our previous simulation we considered that the electric contact thickness is negligible and that there are no losses of light absorption at the front surface of the cell. In reality, this is not totally true. In fact, the surface of the electric contacts tailored inside the cell causes a significant loss of light absorption under illumination. Consider that the contact thickness is $S_{\text {contact }}$ equal to 5

Table 2. Results of impurity concentration optimization.

\begin{tabular}{llllll}
\hline Impurity concentration $\left(\mathrm{cm}^{-3}\right)$ & $10^{15}$ & $10^{16}$ & $10^{17}$ & $10^{18}$ & $10^{19}$ \\
\hline Short-circuit current $\left(\mathrm{mA} / \mathrm{cm}^{2}\right)$ & 47.4 & 46.6 & 46.3 & 46.0 & 37.0 \\
Open circuit voltage (V) & 0.35 & 0.62 & 0.68 & 0.75 & 0.74 \\
Output power $\left(\mathrm{mW} / \mathrm{cm}^{2}\right)$ & 3.40 & 23.7 & 26.9 & 28.7 & 22.6 \\
Fill factor $(\%)$ & 20.83 & 82.86 & 84.27 & 83.36 & 82.56 \\
Efficiency $(\%)$ & 2.53 & 17.44 & 19.75 & 21.09 & 16.61 \\
\hline
\end{tabular}

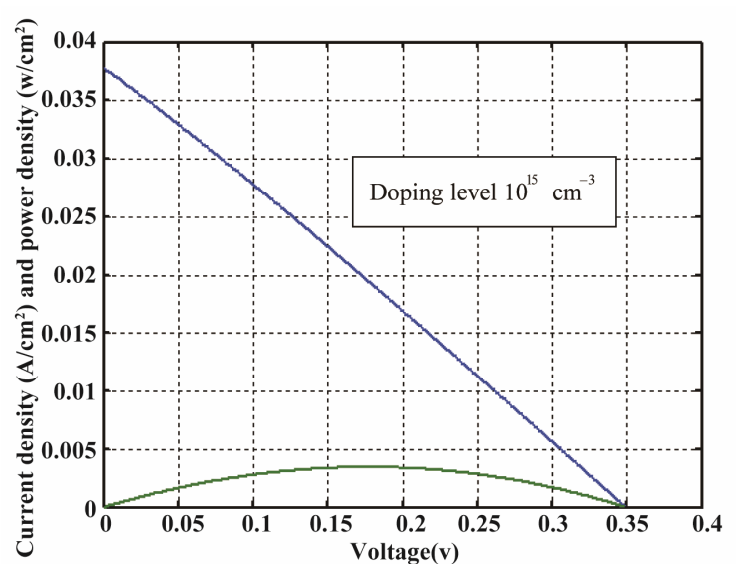

(a) $\mu \mathrm{m}$, which is a realistic value, and that the number of contacts per square centimetre is $k$ equal to 15 . The remaining illuminated surface of the cell will be:

$$
S_{\text {remaining }}\left(\mathrm{cm}^{2}\right)=1-k \cdot S_{\text {contact }}=0.9925
$$

The flux of light received by the photovoltaic cell is:

$$
\varphi_{\text {received }}=\varphi_{\text {total }}\left(1-k \cdot S_{\text {contact }}\right)
$$

We computed our MATLAB program taking in account the received quantum of incident photon energy, and we simulate the efficiency of the n-p-n-p silicon multilayer solar cell for different values of the contact thickness. The program of simulation gives the optimal number of contacts and the optimal distance between contacts giving the maximum efficiency for each value contact thickness.

We use the optimal layers thicknesses obtained in 2.1 and the optimal impurity concentration obtained in 2.2. Figure 6 gives the simulated efficiency versus the contact thickness and the distance between contacts.

We can see from the three dimensional curve, that for each contact thickness correspond an optimal distance between contacts which gives the maximum efficiency of the cell. Table 3 resumes the optimal distance between contacts $\left(d_{\text {contacts }}\right)$ and the maximum efficiency obtained with some choosing values of the contact thickness $S_{\text {con- }}$ tact.

The efficiency of the conceived multilayer solar cell decreases when the thickness of the electric contacts increases. This efficiency drop is due to the non-illuminated surface resulting from the some of electric contact thicknesses. The efficiency of the n-p-n-p silicon solar cell, with a contact thickness of $0.1 \mu \mathrm{m}$ is equal to $20.6 \%$. The efficiency of the cell decreases to only about $15 \%$ with a contact thickness of $5 \mu \mathrm{m}$. Thus, special care may be accorded to the insertion or the growth of the electric contacts to achieve high efficiency values.

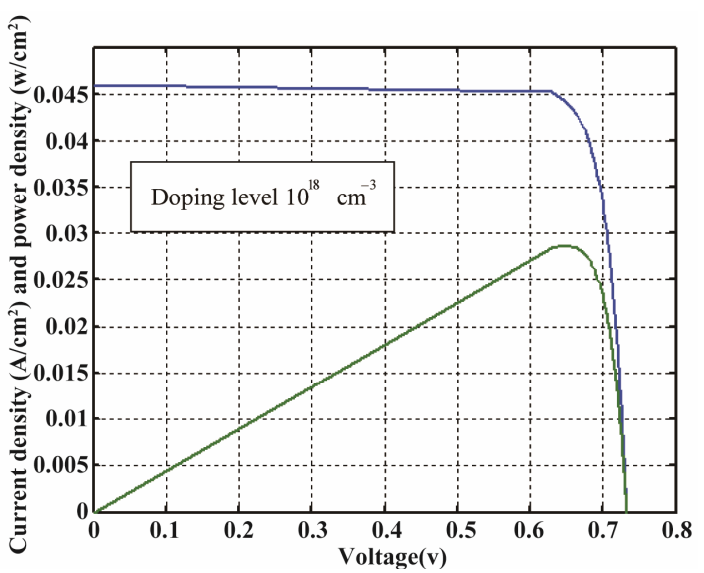

(b)

Figure 5. I-V characteristics of the n-p-n-p silicon solar cell with an impurity concentration of $10^{15} \mathrm{~cm}^{-3}\left(\right.$ a) and $10^{18} \mathrm{~cm}^{-3}(\mathrm{~b})$. 


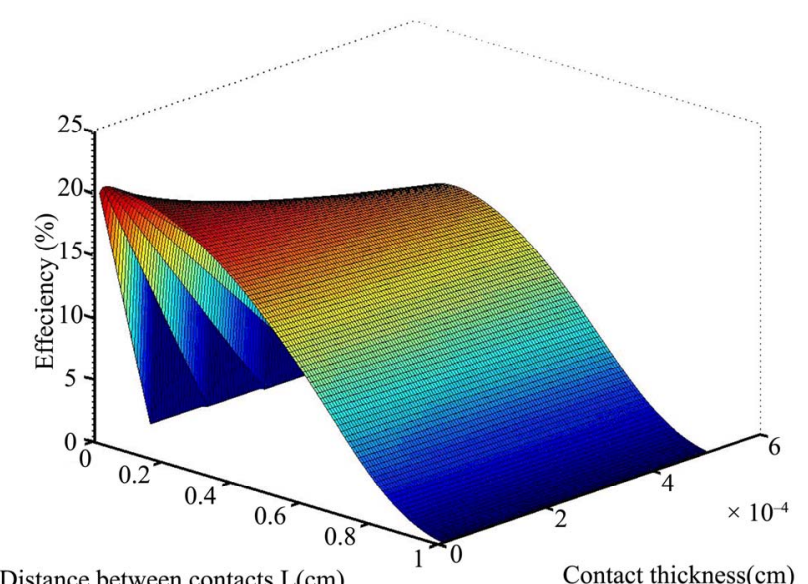

Distance between contacts $\mathrm{L}(\mathrm{cm})$

Contact thickness $(\mathrm{cm})$

Figure 6. Efficiency versus contact thickness and distance between contacts.

Table 3. Optimal efficiency and distance between contacts for different values of contacts surface.

\begin{tabular}{ccccccc}
\hline$S_{\text {contact }}(\mu \mathrm{m})$ & 0.1 & 1 & 1.5 & 2 & 3 & 5 \\
\hline$d_{\text {contacts }}(\mathrm{cm})$ & 0.07 & 0.15 & 0.17 & 0.19 & 0.21 & 0.26 \\
Optimal Efficiency $(\%)$ & 20.6 & 19.0 & 18.4 & 17.8 & 16.9 & 15.3 \\
\hline
\end{tabular}

\section{Conclusions}

In this work we optimized the conception parameters of a proposed n-p-n-p multilayer solar cell composed by four layers of opposite conductivities forming three junctions inside the cell. The electric contacts are tailored vertically to collect the minority carrier generated under illumination.

The optimization of the cell conception was accomplished within three steps: optimization of the layers thicknesses, optimization of the impurity concentration and optimization of the distance between electric contacts. The optimization of the layers thicknesses takes into account the series resistances of each layer which determine the voltage drop inside the cell.

The optimization of the impurity concentration is based on the research of the best compromise between lacks and exceeds of electric carrier to be generated under illumination. Finally the distance between electric contacts was optimized tacking into account the nonilluminated surface due to the contact thicknesses.

The optimal parameters are:

Optimal layers abscissas:

$d_{1}=4 \mu \mathrm{m}$ for the first layer;

$d_{2}=10 \mu \mathrm{m}$ for the second layer;

$d_{3}=45 \mu \mathrm{m}$ for the third one;

The optimal impurity concentration: $10^{18} \mathrm{~cm}^{-3}$;

The optimal electric contact thickness: $0.1 \mu \mathrm{m}$;

The optimal distance between contacts: $0.07 \mathrm{~cm}$.

The optimized photovoltaic structure, with the silicon properties published at the Ioffe institute website [Ioffe], gives an efficiency of $20.66 \%$. The n-p-n-p silicon cell deliver a short circuit current $I_{c c}=45.3 \mathrm{~mA} / \mathrm{cm}^{2}$, an open circuit voltage $V_{o c}=0.746 \mathrm{~V}$ and an output power of 28.5 $\mathrm{mW} / \mathrm{cm}^{2}$. The corresponding fill factor is $F F=84.29 \%$.

These values of the cell performances can be improved by using a best quality of silicon like those used by M. Green in PERC and PERL cells [10] and by using a square based pyramids surface in the front face of the cell.

\section{Acknowledgements}

We are grateful an anonymous referee for helpful comments. We also wish to thank Kim Humphreys for English editing. All errors are ours.

\section{REFERENCES}

[1] A. S. Bouazzi, M. Abaab and B. Rezig, "A New Model of Very High Efficiency Buried Emitter Silicon Solar Cell," Solar Energy Material and Solar Cells, Vol. 46, No. 1, 1997, pp. 29-41. doi:10.1016/S0927-0248(96)00091-8

[2] J. Krc, K. Brecl, F. Smole and M. Topic, "The Effects of Enhanced Light Trapping in Tandem Micromorph Silicon Solar Cells," Solar Energy Materials and Solar Cells, Vol. 90, No. 18-19, 2006, pp. 3339-3344.

[3] J. Kwak, S. Won Kwon and K. S. Lim, "Fabrication of a n-p-p Tunnel Junction for a Protocrystalline Silicon Multilayer/Amorphous Silicon Tandem Solar Cell," Journal of Non-Crystalline Solids, Vol. 352, No. 9-20, 2006, pp. 1847-1850.

[4] R. R. Arya, "Amorphous Silicon Based Solar Cell Technologies: Status, Challenges, and Opportunities," Materials Research Society Symposium Proceedings, Vol. 808 2011, 11 pages. doi:10.1557/PROC-808-A7.5

[5] K. Brecl, F. Smole and J. Furlan, "Modelling of Multilayer Thin-Film Solar Cells," Progress in Photovoltaics: Research and Applications, Vol. 7, No. 6, 1999, pp. 449456.

doi:10.1002/(SICI)1099-159X(199911/12)7:6<449::AIDPIP286>3.0.CO;2-U

[6] A. Bouzidi, A. S. Bouazzi and B. Rezig, "Photocurrent Density Simulation in an n-p-n-p Silicon Multilayer Solar Cell," The European Physical Journal Applied Physics, Vol. 31, No. 1, 2005, pp. 11-16. doi:10.1051/epjap:2005036

[7] A. Bouzidi, H. Hamzaoui, A. S. Bouazzi and B. Rezig, "Analytic Computation of the Photocurrent Density in a n-6H-SiC/p-Si/n-Si/p-Si ${ }_{0.8} \mathrm{Ge}_{0.2}$ Multilayer Solar Cell," Microelectronics Journal, Vol. 37, No. 5, 2006, pp. 388-394. doi:10.1016/i.mejo.2005.06.011

[8] Ioffe Physical Technical Institute, 2012. http://www.ioffe.ru

[9] A. S. Bouazzi, M. Abaab and B. Rezig, "The Effect of the Emitter Resistively on the Characteristics of a Silicon Solar Cell," 14th European Photovoltaic Solar Energy 
Conference, Barcelona, 30 June-4 July 1997.

[10] M. A. Green and S. R. Wenham, "Novel Parallel Multi- junction Solar Cell," Applied Physics Letters, Vol. 65, No. 3, 1994, pp. 2907-2909. doi:10.1063/1.112526 